\title{
Diagnostico da ação do IBAMA na explotação dos recursos pesqueiros no Baixo Amazonas (PA)
}

O Instituto Brasileiro do Meio Ambiente e dos Recursos Naturais Renováveis (IBAMA) tem como objetivo institucional o licenciamento ambiental, o controle da qualidade ambiental, a autorização de uso dos recursos naturais e a fiscalização, monitoramento e controle ambiental. Nesse estudo, são apresentados os resultados das ações do IBAMA na região do Baixo Amazonas (PA), com base nas análises dos autos de infração relacionados a explotação dos recursos pesqueiros, expedidos de 1996 a 2012, nos municípios de Altamira, Itaituba, Juruti, Monte Alegre, Óbidos, Oriximiná, Prainha, Santarém e Vitória do Xingu. Foram emitidos 710 autos de infrações para pessoas físicas e jurídicas, relacionados à falta de registro geral da pesca, licenciamento ambiental e a explotação ilegal de 35 espécies e grupos de espécies de peixes utilizados para o consumo e ornamentação, quelônios e mamíferos aquáticos, que totalizou $\mathrm{R} \$ 4.462 .045,62$ em multas. Altamira, Óbidos e Santarém foram os municípios onde o maior quantitativo e valores em multas foram registradas. A quantidade de infrações e o total anual em multas apresentam tendências de aumento, sendo observado até quaro reincidências no mesmo ano ou em anos distintos. Apesar desses resultados e de sua importância para a conservação dos recursos pesqueiros, podem ser considerados como uma subestimativa, uma vez que o Baixo Amazonas contém 15 municípios em seu perímetro territorial, fator que pode estar relacionados a limitação orçamentária e logística do IBAMA, o que demanda maior atenção por parte do Governo Federal.

Palavras-chave: Atuação; Fiscalização; Pesca.

\section{Diagnosis of IBAMA's action in the exploitation of fishery resources in the Lower Amazon (PA)}

\begin{abstract}
The Brazilian Institute of Environment and Renewable Natural Resources (IBAMA) has as its institutional objective environmental licensing, environmental quality control, authorization of the use of natural resources and environmental monitoring, monitoring and control. In this study, the results of IBAMA's actions in the Lower Amazon region (PA) are presented, based on the analysis of the infraction notices related to the exploitation of fishing resources, issued from 1996 to 2012 , in the municipalities of Altamira, Itaituba, Juruti, Monte Alegre, Óbidos, Oriximiná, Prainha, Santarém and Vitória do Xingu. 710 notices of infraction were issued to individuals and legal entities related to the lack of general fishing records, environmental licensing and illegal exploitation of 35 species and groups of fish species used for consumption and ornamentation, turtles and aquatic mammals, which totaled $R \$ 4,462,045.62$ in fines. Altamira, Óbidos and Santarém were the municipalities where the highest amount and fines were recorded. The amount of infractions and the annual total in fines tend to increase, being observed up to four recurrences in the same year or in different years. Despite these results and their importance for the conservation of fishery resources, they can be considered as an underestimate, since the Lower Amazon contains 15 municipalities in its territorial perimeter, a factor that may be related to IBAMA's budget and logistical limitation, which may demands greater attention from the Federal Government.
\end{abstract}

Keywords: Acting; Supervision; Fishing.

Topic: Desenvolvimento, Sustentabilidade e Meio Ambiente

Reviewed anonymously in the process of blind peer

Charles Hanry Faria Junior

Instituição de Ensino Superior, País

http://orcid.org/0000-0002-2124-1351

charlesufopa@gmail.com

Jordson de Souza e Souza (iD

Universidade Federal do Oeste do Pará, Brasil

http://lattes.cnpq.br/7571403046809712

http://orcid.org/0000-0003-2377-4263

jordsonssouza@gmail.com

Thiago Marinho Pereira (it)

Universidade Federal do Oeste do Pará, Brasi

http://lattes.cnpq.br/5204637707676338

http://orcid.org/0000-0002-4340-0130

tmarinhopereira@gmail.com

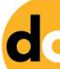

DOI: 10.6008/CBPC2179-6858.2018.006.0026
Received: 22/08/2018

Approved: 27/08/2018
Referencing this:

FARIA JUNIOR, C. H.; SOUZA, J. S.; PEREIRA, T. M.. Diagnostico da ação do IBAMA na explotação dos recursos pesqueiros no Baixo Amazonas (PA). Revista Ibero-Americana de Ciências Ambientais, v.9, n.6, p.273-282, 2018. DOI: http://doi.org/10.6008/CBPC2179$\underline{6858.2018 .006 .0026}$ 


\section{INTRODUÇÃO}

O Instituto Brasileiro de Meio Ambiente e dos Recursos Renováveis (IBAMA) foi fundando em 22 de fevereiro de 1989, com a promulgação da Lei no 7.735 , com atribuições para: exercer o poder de polícia ambiental; executar ações das políticas nacionais de meio ambiente, referentes às atribuições federais, relativas ao licenciamento ambiental, ao controle da qualidade ambiental, à autorização de uso dos recursos naturais e à fiscalização, monitoramento e controle ambiental; e executar as ações supletivas de competência da União de conformidade com a legislação ambiental vigente, conforme a Lei no 11.516, de 28 de agosto de 2007 (IBAMA, 2012).

No contexto dos recursos pesqueiros, a Lei Federal № 7.679/1988, que proíbe a pesca nos cursos d'água, águas paradas ou mar territorial nacional durante os períodos de desova, reprodução ou de defeso, ficou sob a competência do IBAMA, que passou a fixar os períodos de proteção reprodutiva e as espécies protegidas pela normativa, de acordo com as peculiaridades de cada região.

Dessa forma, o IBAMA emite anualmente portarias delimitando as estações do ano em que se permite a pesca, emite as licenças de pesca, delimita as restrições aos equipamentos e insumos autorizados nas pescarias, bem como as cotas de captura. Além disso, compete ao IBAMA o controle e a fiscalização das medidas estabelecidas pela política de ordenamento pesqueiro, com o objetivo de garantir a exploração racional dos recursos pesqueiros, de acordo com as normas e regulamentações estabelecidas por este órgão, na busca da sustentabilidade (MAIA, 2009).

Assim, a partir de sua criação, o IBAMA vem fiscalizando e reprimindo os crimes ambientais, emitindo autos de infração, determinando multas aos infratores, apreendendo o produto da infração e os instrumentos/equipamentos utilizados, colaborando para a conservação e a preservação ambiental. 0 Estado do Pará, na ótica dos recursos pesqueiros, tem destaque como o principal produtor Nacional, contribuindo com $18 \%$ da produção brasileira (IBAMA, 2004).

Entre as sub-regiões Paraenses, o Baixo Amazonas contribui com $30 \%$ da produção estadual (ISAAC et al., 1996; ALMEIDA et al., 2001), o que mostra a necessidade da atenção especial das ações fiscalizatórias do IBAMA sobre essa região. Nesse sentido, o presente trabalho apresenta o resultado das autuações do IBAMA na Região do Baixo Amazonas (PA), sob a ótica das infrações registradas, espécies relacionadas nos autos de infração emitidos e o impacto econômico sobre os infratores.

\section{METODOLOGIA}

A Região do Baixo Amazonas (PA) foi selecionada como área de estudo por sua importância na produção pesqueiros do Estado e a existência da Gerência Executiva do IBAMA na cidade de Santarém, localizada na Avenida Tapajós, no 2.267, Laguinho. A partir de contatos institucionais, foi possível obter listagem em PDF dos autos de infrações relacionadas aos organismos aquáticos, resultantes das ações de fiscalização na região do Baixo Amazonas emitidas de 1996 a 2012.

Entre as informações contidas nos autos constam os municípios de origem, as datas e tipo das infrações cometidas, a descrição da infração, as espécies envolvidas, seus quantitativos e o valor resultante 
das multas emitidas ao longo da escala temporal estudada. Nas análises foram utilizadas ferramentas da estatística descritiva, além do emprego de análise de regressão linear simples para representar a tendência de mudança no quantitativo de multas aplicadas e o valor resultante ao longo dos anos de estudos (ZAR, 1999).

\section{RESULTADOS}

Os autos obtidos descrevem 710 infrações registradas em nove municípios da Região do Baixo Amazonas - Pará entre janeiro de 1996 a maio de 2012, aplicadas a pessoas físicas e jurídicas que explotam, beneficiam e comercializam organismos aquáticos nessa região, conforme se ode verificar na tabela 1, que foi denominada 'Quantitativo e somatória das infrações registrados pelo IBAMA por município do Baixo Amazonas (PA)'.

Tabela 1: Quantitativo e somatória das infrações registrados pelo IBAMA por município do Baixo Amazonas (PA).

\begin{tabular}{|c|c|c|}
\hline Municípios & No de Infrações & Valor das Multas \\
\hline Altamira & 62 & $\mathrm{R} \$ 1.063 .192,00$ \\
\hline Itaituba & 33 & $\mathrm{R} \$ 97.773,00$ \\
\hline Juruti & 30 & $\mathrm{R} \$ 322.161,70$ \\
\hline Monte Alegre & 43 & $\mathrm{R} 265.113,97$ \\
\hline Óbidos & 145 & $\mathrm{R} \$ 397.743,35$ \\
\hline Oriximiná & 85 & $\mathrm{R} \$ 116.972,10$ \\
\hline Prainha & 9 & $\mathrm{R} 116.160,00$ \\
\hline Santarém & 295 & $\mathrm{R} \$ 2.065 .879,50$ \\
\hline Vitória do Xingu & 8 & $\mathrm{R} \$ 17.050,00$ \\
\hline TOTAL & $\mathbf{7 1 0}$ & $\mathbf{R} \mathbf{4 . 4 6 2 . 0 4 5 , 6 2}$ \\
\hline
\end{tabular}

Dos $R \$ 4.462 .045,62$ que totalizam o montante das infrações, $86,24 \%$ envolvem a constatação da captura de organismos aquáticos protegidos por normativas (tamanho mínimo de captura, pesca e comercialização proibida, defeso reprodutivo), com destaque para o pescado direcionado ao consumo humano, que corresponde a 98,46\% das infrações (tabela 2).

Tabela 2: Organismos aquáticos por finalidade de utilização listada nas infrações registradas pelo IBAMA na Região do Baixo Amazonas (PA).

\begin{tabular}{|c|c|c|c|}
\hline Organismo & Finalidade & Quantitativo & Unidade \\
\hline \multirow{2}{*}{ Peixes } & Consumo & 200.960 & $\mathrm{Kg}$ \\
\hline & Ornamental & 3.298 & Indivíduo \\
\hline Quelônios & Consumo & 44 & Indivíduo \\
\hline Mamíferos aquáticos & Consumo & 2 & Indivíduo \\
\hline
\end{tabular}

As infrações emitidas foram divididas em 6 grupos (tabela 3), onde a cadeia de produção e comercialização tem destaque, correspondendo a 71,65\% das infrações, seguida da pesca com técnicas, local ou período proibido (16,56\%). Entre os organismos aquáticos relacionados nas infrações são listados peixes, quelônios e mamíferos aquáticos, totalizando 35 denominações que podem superar 150 espécies (duas ou mais espécies para o mesmo nome vulgar) de quelônios e peixes utilizados para o consumo ou ornamentais. Quando relacionadas com os valores das multas, as três espécies que apresentaram o maior valor acumulado foram: a piramutaba (Brachyplatystoma vaillantii), os cascudos (Loricariidae) e os maparás (Hypophthalmus spp.), como se pode ver na tabela 4. 
Tabela 3: Descrição e valor envolvido nas multas aplicadas aos atores que capturam, beneficiam e comercializam organismos aquáticos nos municípios do Baixo Amazonas (PA).

\begin{tabular}{|c|c|c|}
\hline Descrição & Frequência & Valor das Multas \\
\hline $\begin{array}{l}\text { Adquirir, armazenar, transportar, beneficiar, comercializar, industrializar ou exportar peixe } \\
\text { abaixo do tamanho mínimo, proveniente de pesca proibida (defeso) ou preservada. }\end{array}$ & 420 & $R \$ 3.196 .125,15$ \\
\hline Captura de peixe boi. & 2 & $\mathrm{R} \$ 1.900,00$ \\
\hline Comercializar ou transportar tartaruga sem licença. & 3 & $\mathrm{R} \$ 868,00$ \\
\hline $\begin{array}{l}\text { Ausência ou perda da validade do registro (pescador, embarcação, unidade de } \\
\text { beneficiamento e estocagem ou indústria). }\end{array}$ & 37 & $\mathrm{R} \$ 32.030,00$ \\
\hline Pescar com técnicas, local ou período proibido. & 226 & $\mathrm{R} \$ 739.092,47$ \\
\hline Pescar quantidade superior à permitida. & 22 & $\mathrm{R} \$ 492.030,00$ \\
\hline TOTAL & 710 & $\mathrm{R} \$ 4.462 .045,62$ \\
\hline
\end{tabular}

Tabela 4: Descrição quantitativa (kg e unidade - und) e qualitativa do grupo de espécies e espécies, frequência de ocorrência e valor da multa estabelecida nos autos de infração do IBAMA no Baixo Amazonas (PA).

\begin{tabular}{|c|c|c|c|c|c|}
\hline Organismo & Finalidade & Denominação & Nome científico & Quantidade & Valor da multa \\
\hline \multirow{33}{*}{ Peixes } & \multirow{27}{*}{ Consumo } & Acará & Cichlidae & $11 \mathrm{~kg}$ & $\mathrm{R} \$ 955,71$ \\
\hline & & Acari & Loricariidae & $776 \mathrm{~kg}$ & $\mathrm{R} \$ 25.495,00$ \\
\hline & & Aracu & Anostomidae & $2.640 \mathrm{~kg}$ & $\mathrm{R} \$ 41.189,17$ \\
\hline & & Arraia & Potamotrygons spp. & $179 \mathrm{~kg}$ & $\mathrm{R} \$ 118.710,00$ \\
\hline & & Branquinha & Curimatidae & $907 \mathrm{~kg}$ & $\mathrm{R} \$ 3.631,67$ \\
\hline & & Cachorra & Cynodontidae & $76 \mathrm{~kg}$ & $\mathrm{R} \$ 1.955,71$ \\
\hline & & Cujuba & Doradidae & $28 \mathrm{~kg}$ & $\mathrm{R} \$ 1.101,67$ \\
\hline & & Curimatá & Prochilodus nigricans & $4.575 \mathrm{~kg}$ & $\mathrm{R} \$ 201.447,38$ \\
\hline & & Dourada & Brachyplatystoma rousseauxii & $238 \mathrm{~kg}$ & $\mathrm{R} \$ 402.550,00$ \\
\hline & & Filhote & Pimelodidae & $4 \mathrm{~kg}$ & $\mathrm{R} \$ 323,33$ \\
\hline & & Fura-calça & Pimelodina flavipinnis & $1.566 \mathrm{~kg}$ & $\mathrm{R} \$ 22.980,71$ \\
\hline & & Jacundá & Crenicichla spp. & $200 \mathrm{~kg}$ & $\mathrm{R} \$ 51.750,00$ \\
\hline & & Jaraqui & Semaprochilodus sp. & $100 \mathrm{~kg}$ & $\mathrm{R} \$ 134,00$ \\
\hline & & Jatuarana & Characidae & $130 \mathrm{~kg}$ & $R \$ 2.000,00$ \\
\hline & & Mandubé & Ageneiosus brevifilis & $3 \mathrm{~kg}$ & $\mathrm{R} \$ 165,00$ \\
\hline & & Mapará & Hypophthalmus spp. & $58.434 \mathrm{~kg}$ & $\mathrm{R} \$ 568.004,05$ \\
\hline & & Pacu & Mylossoma spp. & $6.168 \mathrm{~kg}$ & $\mathrm{R} \$ 110.617,38$ \\
\hline & & Peixe* & Animalia & $65.220 \mathrm{~kg}$ & $\mathrm{R} \$ 585.820,75$ \\
\hline & & Pescada & Plagioscion spp. & $165 \mathrm{~kg}$ & $\mathrm{R} \$ 2.413,21$ \\
\hline & & Piramutaba & Brachyplatystoma vaillantii & $35.007 \mathrm{~kg}$ & $\mathrm{R} \$ 645.190,00$ \\
\hline & & Piranha & Serrasalmidae & $23 \mathrm{~kg}$ & $\mathrm{R} \$ 765,00$ \\
\hline & & Pirapitinga & Piaraetus braehypomus & $103 \mathrm{~kg}$ & $\mathrm{R} \$ 2.909,17$ \\
\hline & & Pirarucu & Arapaima gigas & $14.640 \mathrm{~kg}$ & $\mathrm{R} \$ 78.309,72$ \\
\hline & & Surubim & Pseudoplatystoma spp. & $1.834 \mathrm{~kg}$ & $\mathrm{R} \$ 109.063,75$ \\
\hline & & Tambaqui & Colossoma macropomum & $7.673 \mathrm{~kg}$ & $\mathrm{R} \$ 266.287,72$ \\
\hline & & Traíra & Erythrinidae & $70 \mathrm{~kg}$ & $\mathrm{R} \$ 1.750,00$ \\
\hline & & Tucunaré & Cichilidae & $190 \mathrm{~kg}$ & $\mathrm{R} \$ 5.195,00$ \\
\hline & \multirow{6}{*}{ Ornamental } & Cascudo & Loricariidae & 1.053 und & $\mathrm{R} \$ 568.100,01$ \\
\hline & & Cará de pão & Panaque spp. & 444 und & $\mathrm{R} \$ 11.140,00$ \\
\hline & & Cascudo & Panaque cf. nigrolineatus & 100 und & $\mathrm{R} \$ 1.000,00$ \\
\hline & & Acari borracha, bola azul & Parancistrus spp. & 835 und & $\mathrm{R} \$ 750,00$ \\
\hline & & Cascudo & Peckoltia spp. & 200 und & $\mathrm{R} \$ 2.000,00$ \\
\hline & & Picota ouro ou cutia & Scobiancistrus spp. & 666 und & $\mathrm{R} \$ 11.140,00$ \\
\hline Mamífero aquático & Consumo & Peixe Boi & Trichechus manatus & 2 und & $\mathrm{R} \$ 1.900,00$ \\
\hline Quelônios & Consumo & Tartaruga & Podocnemis spp. & 44 und & $\mathrm{R} \$ 1.218,00$ \\
\hline
\end{tabular}

Legenda: *Espécies não identificadas.

Entre o pescado direcionado ao consumo humano, os maparás, os pacus, a curimatã, o tambaqui e o surubim foram os mais frequentes, uma vez que têm a preferência popular, os maiores valores de comercialização e atratividade para as indústrias de beneficiamento de pescado localizadas na região, conforme ilustra a figura 1. 


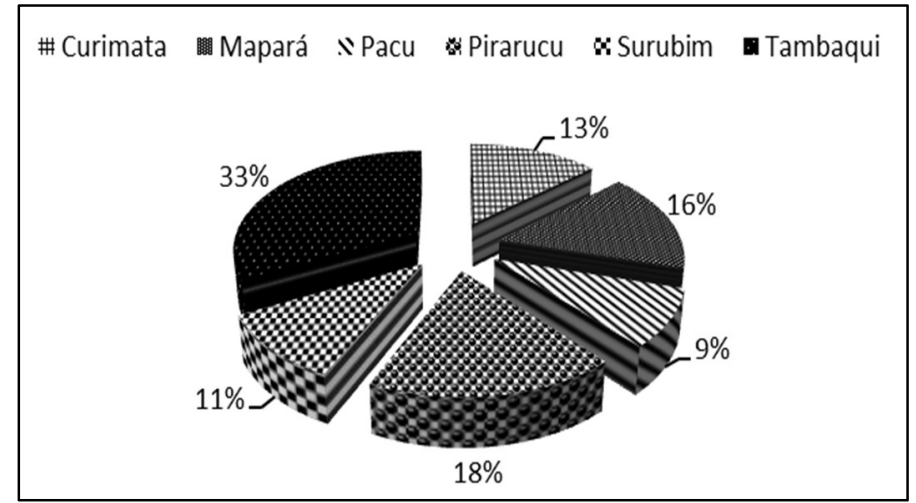

Figura 1: Principais espécies ou grupo de espécies (duas ou mais espécies, com a mesma denominação) listadas nos autos de infração aplicadas pelo IBAMA na região este do Pará.

Ao relacionarmos as multas com os anos estudados, observamos que, tanto a quantidade de infrações, quanto a somatória dos valores anuais apresentam tendências de aumento (figura 2). 0 ano de 2012 não foi inserido nessa análise por conter dados apenas até o mês de maio, o que influenciaria negativamente no resultado.

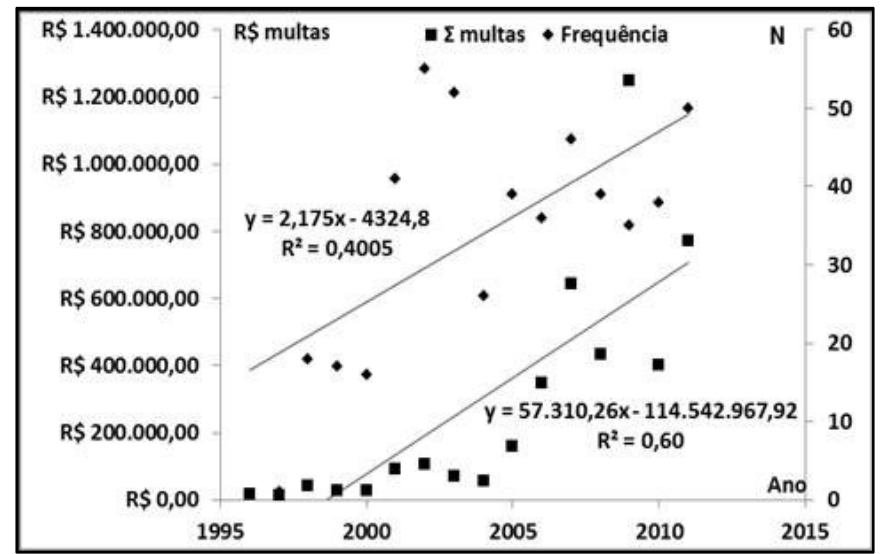

Figura 2: Variação anual do número de infrações aplicadas pelo IBAMA nos municípios do Baixo Amazonas (PA) e valor resultante.

Independente da categoria, os infratores em 7,46\% dos casos são reincidentes em até quatro vezes, flagrados duas os mais vezes no mesmo ano ou ao longo de três anos diferentes, além de atuarem em mais de um município na tentativa de permanecer na prática proibida ou evitar a ação do IBAMA. Para finalizar, de forma a demonstrar o risco assumido pelos infratores reincidentes ou não (só aparecem uma vez na lista dos infratores), foi determinado que o faturamento que poderiam auferir com a venda do produto apreendido (calculado sobre o valor estimado de mercado para a primeira comercialização), comparado ao montante resultante das multas (tabela 5), resulta em um prejuízo superior a três vezes o valor que poderiam obter. Porém de forma individualizada por município, essa diferença tem amplitude de 1,04 a 24,60 vezes o valor da multa em relação ao faturamento.

Tabela 5: Estimativa de faturamento com base na produção apreendida e valor correspondente da multa por município.

\begin{tabular}{|c|c|c|c|}
\hline Município & Estimativa de faturamento & Soma das multas & Soma das multas/ Estimativa de faturamento \\
\hline Altamira & $\mathrm{R} \$ 43.215,00$ & $\mathrm{R} \$ 1.063 .192,00$ & 24,60 \\
\hline Itaituba & $\mathrm{R} \$ 21.381,00$ & $\mathrm{R} \$ 97.773,00$ & 4,57 \\
\hline Juruti & $\mathrm{R} \$ 104.720,00$ & $\mathrm{R} \$ 322.161,70$ & 3,08 \\
\hline Monte Alegre & $\mathrm{R} \$ 99.114,00$ & $\mathrm{R} \$ 265.113,97$ & 2,67 \\
\hline
\end{tabular}




\begin{tabular}{|c|c|c|c|}
\hline Óbidos & $\mathrm{R} \$ 368.633,00$ & $\mathrm{R} \$ 397.743,35$ & 1,08 \\
\hline Oriximiná & $\mathrm{R} \$ 30.531,00$ & $\mathrm{R} \$ 116.972,10$ & 3,83 \\
\hline Prainha & $\mathrm{R} \$ 111.680,00$ & $\mathrm{R} \$ 116.160,00$ & 1,04 \\
\hline Santarém & $\mathrm{R} \$ \mathbf{6 0 6 . 8 7 9 , 0 0}$ & $\mathrm{R} \$ 2.065 .879,50$ & 3,40 \\
\hline Vitória do Xingu & $\mathrm{R} \$ 14.628,00$ & $\mathrm{R} \$ 17.050,00$ & 1,17 \\
\hline Total & $\mathrm{R} \$ \mathbf{1 . 4 0 0 . 7 8 1 , 0 0}$ & $\mathrm{R} \$ \mathbf{4 . 4 6 2 . 0 4 5 , 6 2}$ & $\mathbf{3 , 1 9}$ \\
\hline
\end{tabular}

\section{DISCUSSÃO}

A explotação da riqueza íctia Amazônica é descrita por inúmeros autores como uma atividade de importância social, cultural e econômica, praticada por diferentes categorias de pescadores, em diversificados ambientes aquáticos, mediante o emprego de uma ampla variedade de aparelhos de captura e técnicas de pesca, que resulta em uma produção multiespecífica desembarcada nos mercados regionais, estaduais e internacionais (PETRERE, 1978; BARTHEM et al., 1992; CERDEIRA et al., 1997; BATISTA et al., 1998; RUFFINO, 2000; CARDOSO et al., 2004; FARIA-JUNIOR et al., 2006; BRASIL, 2012; NAKAÚTH et al., 2012; FARIA-JUNIOR, 2013; COELHO et al., 2017; CARDOSO et al., 2017; BRELAZ et al., 2018).

Porém, no processo histórico de explotação do recurso pesqueiro, os estímulos públicos para a modernização do seguimento pesqueiro e o maior aproveitamento do recurso, aliada ao aumento da demanda por proteína de origem pesqueira, proporcionaram a intensificação da pesca, principalmente para espécies de maior preferência popular e valor comercial, como o pirarucu e o tambaqui, que apresentaram sinais de sobrepesa, demandando medidas de ordenamento das capturas com vista a sua conservação (MCGRATH et al., 1993; ISAAC et al., 1995; CRAMPTON, 1999; DIAS et al., 2013).

Na tentativa de promover o ordenamento setorial para o uso sustentável dos recursos e à fiscalização da atividade, o Governo Federal, ao longo dos anos, criou diversos órgãos federais, como a Superintendência do Desenvolvimento da Pesca (SUDEPE), o Ministério do Meio Ambiente (MMA), o IBAMA, a Secretaria Especial de Aquicultura e Pesca (SEAP) e o Ministério da Pesca e Aquicultura (MPA) (SÃO PAULO, 2012).

O IBAMA, a partir de 1989, passou a desenvolver várias ações de combate aos crimes ambientais (IBAMA, 2008), como as apresentadas no presente estudo, que resultaram em um quantitativo expressivo de autos de infração emitidos, que totalizaram um valor significativo em multas relacionadas a pesca ilegal, a falta de registro de pescador profissional e ausência do licenciamento nas diferentes esferas desse seguimento produtivo na Região do Baixo Amazonas (PA). Santarém e Óbidos foram os municípios de origem do maior quantitativo de multas expedidas, entretanto, em termos econômicos, Santarém e Altamira têm destaque, com os maiores montantes em multas.

O que pode explicar o motivo pelo qual o município do Óbidos detém essa posição no ranking de multas aplicadas pelo IBAMA, pode se relacionar a vocação do município para a produção de mapará, grupo de espécie que, nos anos de 2001, 2002, 2003 e 2004, liderou o desembarque no município (IBAMA, 2001, 2002, 2003; THOMÉ-SOUZA, 2007; ISSAC et al., 2008). Essa tendência pode ter continuidade nos demais anos relacionados no período da ação do IBAMA estudado, porém, sem informações encontradas devido à falta de estudos locais, uma vez que os boletins estatísticos passaram a relacionar a produção por região e não por município. 
Outro motivado pode ter relação com o quantitativo de pescadores. Óbidos congrega 5.730 pescadores cadastrados em sua Colônia de Pescadores, porém, foram estimados em 7.520 segundo o Movimento dos Pescadores do Baixo Amazonas (CAMPOS et al., 2007; ISAAC et al., 2008). Assim, existe a possibilidade de que, em algum momento, parte do contingente não estivesse de posse do seu Registro Geral da Pesca - RGP, ou estar com o mesmo vencido, ou não o possuir quando da abordagem do IBAMA, uma vez que é obrigatório (Decreto-Lei no 221, de 28 de fevereiro de 1967 e ratificado pela Lei no 11.959, de 26 de junho de 2009).

Em Santarém, o mapará também têm registros como grupo de espécies mais desembarcadas nos anos de 2001, 2003 e 2004, e entre as 10 mais desembarcadas em 2002 (RUFFINO, 2001, 2002, 2003; THOMÉSOUZA, 2007; ISSAC et al., 2008), além de congregar o maior quantitativo populacional (IBGE, 2010) e quantitativo de pescadores da Região do Baixo Amazonas, com registros de 9.837 pescadores na Colônia de Pescadores (RUFFINO et al., 1994; CAMPOS et al., 2007). Além de se destacar como o principal porto de desembarque pesqueiro (RUFFINO et al., 2002, 2005, 2006) e onde, segundo Almeida et al. (2002), Isaac et al. (2004) e Almeida (2006), a metade do pescado é direcionado aos frigoríficos (indústrias de beneficiamento de pescado), com destaque para os bagres.

Os munícios de Óbidos e Santarém, de forma mais abrangente, além do mapará, explotam espécies como o acarí-bodó, os aracus, as arraias, a curimatã, o fura-calça, os pacus, a piramutaba, o pirarucu, o surubim e o tambaqui, que são protegidas por Portarias e Instrução Normativa como: Portaria IBAMA no 08 de 02/02/1996, Portaria no 001 de 02.02.1996 SUPES/PA, Instrução Normativa MMA no 13, de 14/10/2004, Instrução Normativa № 34, de 18/06/2004, Portaria IBAMA no 48 de 05/11/2007, que descumpridas, resultarão em multas.

O fato do grupo de infrações com maior frequência de ocorrência abranger o período de defeso reprodutivo (30\% das infrações) mostra a importância que deve ser dada as ações de repressão ao ilícito, com a finalidade de proteger os organismos aquáticos durante a fase crítica de seu ciclo de vida, evitando a pesca no momento em que os peixes estão mais vulneráveis à captura (se agrupam formando grande cardumes entre os meses de outubro a fevereiro), favorecendo a sustentabilidade no uso dos estoques pesqueiros (BRASIL, 2012).

$\mathrm{Na}$ ótica da explotação dos peixes ornamentais, o município de Altamira tem destaque como um dos maiores produtores do Estado, com produção quantificada em 198.000 exemplares no ano de 1997; 197.729 em 2001; 269.424 em 2003; 655.137 em 2004; e 261.237 em 2005. Nesse seguimento produtivo, atua um quantitativo de pescadores incerto, com estimativas 1.500 em 2001, que direcionam a produção de peixes ornamentais para 10 empresas, onde se destacam os loricarídeos (principal grupo de espécies relacionadas nos autos de infração, identificados apenas pelo gênero), que representaram aproximadamente $80 \%$ dos indivíduos comercializados (PAULA, 2007; CAMARGO et al., 2012).

Santarém produziu, em 2005, um quantitativo de 706.001 exemplares de peixes ornamentais (PAULA, 2007). É conhecida como local de comercialização da produção de outros municípios e rota para o deslocamento da produção para o mercado nacional e internacional, onde inúmeras apreensões foram 
realizadas. Dessa forma, o quantitativo total em multas para esse município não reflete necessariamente ilícitos locais, e sim o resultado da ação externa, não havendo, entretanto, mecanismos de diferenciação.

No contexto geral das infrações direcionadas para a produção de organismos aquáticos para fins ornamentais, foram observadas apreensões somente para os municípios de Altamira (60,27\%), Santarém $(33,65 \%)$ e Itaituba (6,08\%), reconhecidas áreas de produção e comercialização de 715.931 exemplares em 2006, movimentando entre $\mathrm{R} \$ 430.754,00$ a $\mathrm{R} \$ 4.000 .000 /$ ano somente no primeiro elo da cadeia produção e comercialização (ISAAC, 2008), o que confirma a importância econômica desse segmento e a necessidade de monitoramento por arte do IBAMA.

Os resultados mostram que não só os pescadores, armadores (proprietários dos meios de produção utilizados na pesca), intermediários (atravessadores que compram e revendem a produção), empresários e demais atores envolvidos na cadeia produtiva da pesca, incluindo os consumidores, desconhecem ou desconsideram em algum momento a regulamentação, o que evidencia a necessidade das ações do IBAMA no âmbito setor pesqueiro, principalmente quando se observa que tanto a quantidade de infrações quanto a somatória dos valores anuais apresentam tendências de aumento, apesar do montante em multas aplicadas ser 3,19 vezes superior ao valor que os infratores poderiam obter com a venda da produção apreendida (caso comercializada).

O que aparentemente dá uma ideia que o risco em praticar o ilícito é compensatório (SCHMITT, 2015; ARAÚJO, 2017), uma vez que foram observadas reincidências tanto para pessoas físicas como jurídicas. Para agravar esse cenário, Araújo (2017) destaca que somente 4\% dos valores das multas são efetivamente arrecadados como resultado da perda de validade do período de cobrança (caducam) devido ao excesso de prazo para recursos, inércia na execução e judicialização, o que resulta na perda de arrecadação, que atualizada a partir dos valores obtidos em multa para o presente estudo $(R \$ 4.462 .045,62)$, pelo Índice Geral de Preços do Mercado - IGPM, alcançaria a cifra de R\$8.255.344,47.

Apesar dessa realidade, o fato da Região do Baixo Amazonas conter 15 municípios (IBGE, 2017) e os resultados do presente trabalho abordarem apenas 9, leva a considerá-los como uma subestimativa das irregularidades que ocorrem, o que demanda maior investimento por parte do poder público para ampliar as ações fiscalizadoras e contribuir efetivamente para a conservação dos recursos pesqueiros, vital para a manutenção econômica dos atores envolvidos na cadeia de produção e comercialização, bem como na garantia da oferta de pescado a população do Baixo Amazonas.

A correção dessa limitação só será possível com um maior aporte orçamentário do governo federal para prover a infraestrutura ideal ao controle do uso sustentável dos recursos naturais (IBAMA, 2008; PAULO JÚNIOR et al., 2012), aliada a ações de conscientização da classe produtiva e de educação ambiental aos consumidores, para difundir a importância do respeito as normas reguladoras da exploração dos recursos pesqueiros e a manutenção dos ecossistemas aquáticos (LONGUINE et al., 2011). 


\section{CONCLUSÕES}

As ações de fiscalização do IBAMA relacionadas ao uso dos recursos pesqueiros na Região do Baixo Amazonas resultaram em um elevado quantitativo e valores em multas, contribuindo na repressão do uso ilegal e desordenado dos recursos pesqueiros regionais, entretanto, as limitações orçamentárias e logísticas impedem uma ação mais abrangente do IBAMA, demandando maior atenção por parte do Governo Federal, com vistas a manutenção da pesca, atividade de importância social, cultural e econômica reconhecida na Amazônia.

\section{REFERÊNCIAS}

ALMEIDA, O. T.. A indústria pesqueira na Amazônia. Manaus: IBAMA, 2006.

ALMEIDA, O.; LORENZEN, K.; MCGRATH, D. G.. Impact of Comanagement regimes on the exploitation and productivity of the floodplain lake fisheries in the Lower Amazon. In: PROCEEDING OF THE INTERNATIONAL ASSOCIATION FOR THE STUDY OF COMMON PROPERTY. Anais. Harare: 2002.

ALMEIDA, O.; MCGRATH, D. G.; RUFFINO M. L.. The commercial fisheries of the lower Amazon: an economic analysis. Fisheries Management and Ecology, v.8, p.253269, 2001.

ARAÚJO, S. M. V. G.. Desafios do IBAMA para a gestão ambiental no Brasil. Brasília: IPEA, 2017.

BARTHEM, R. B.; PETRERE, J. R.; ISAAC, V.; RIBEIRO, M. C. L.; MCGRATH, D.; VIEIRA, I. J. A.; BARCO, M. V.. A pesca na Amazônia: problemas e perspectivas para o seu manejo. In: SEMINÁRIO SOBRE 'MANEJO DA VIDA SILVESTRE'. Anais. Belém: CCC, 1992.

BRASIL. Ministério da Pesca e Aquicutura. Boletim Estatístico da Pesca e Aquicultura. Brasília: MPA, 2012.

BRELAZ, R. L.; FARIA-JUNIOR, C. H.; RIBEIRO, F. R. V.. Caracterização da atividade pesqueira na comunidade Vila Flexal do município de Óbidos, Pará, Brasil: subsídios para gestão dos recursos. Scientia Amazonia, v.7, n.1, p.134-155, 2018.

CAMARGO, M.; CARVALHO JÚNIOR, J.; ESTUPIÑAN, R. A.. Peixes comerciais da ecorregião aquática Xingu-Tapajós. Brasília: CETEM, 2012.

CAMPOS, J. R.; FERREIRA, L. V.; APEL, M.; PEREIRA, S. M. L.. Diagnóstico Regional: os pescadores e a pesca na região oeste do Pará e Baixo Amazonas. Santarém: MOPEBAM, 2007.

CARDOSO, R. S.; BATISTA, V. S.; FARIA-JÚNIOR, C. H.; MARTINS, W. R.. Aspectos econômicos e operacionais das viagens da frota pesqueira de Manaus, Amazônia Central. Acta Amazonica, v.34, n.2, p.301-307, 2004.

CARDOSO, R. S.; FARIA-JUNIOR, C. H.. Análise econômica das pescarias em canoas motorizadas no município de Parintins, região do Baixo rio Amazonas, Brasil. Scientia Amazonia, v.6, n.3, p.58-68, 2017.
COELHO, A. C. S.; FARIA-JUNIOR, C. H.; SOUSA, K. N. S. Fatores que influenciam a compra de peixes por classe social no município de Santarém-PA. Agroecossistemas, v.9, n.1, p.62-83, 2017.

DIAS, G. A. C.; BARBOZA, R. S. L.; DIAS JÚNIOR, B. F.; BRITO, D. M. C.; DIAS, T. C. A. C.. Diagnóstico da pesca ilegal no Estado do Amapá, Brasil. Revista Internacional de Direito Ambiental e Políticas Públicas, n.5, p.43-58, 2013.

FARIA-JUNIOR, C. H.. Avaliação da rentabilidade da pesca comercial artesanal e primeira comercialização do pescado no estado do Amazonas, Brasil. Tese (Doutorado em Ciências Pesqueiras nos Trópicos) - Universidade Federal do Amazonas, Manaus, 2013.

FARIA-JÚNIOR, C. H.; BATISTA, V. S.. Repartição da renda derivada da primeira comercialização do pescado na pesca comercial artesanal que abastece Manaus (Estado do Amazonas, Brasil). Acta Scientarum Human and Social Sciences, Maringá, v.28, n.1, p.131-136, 2006. DOI: http://doi.org/10.4025/actascihumansoc.v28i1.190

IBAMA. Instituto Brasileiro do Meio Ambiente e dos Recursos Naturais Renováveis. Uma janela para a informação ambiental. Revista do IBAMA, v.2, n.3, 2008.

IBGE. Instituto Brasileiro de Geografia e Estatística. Contagem da população em 2010. Brasília: IBGE, 2012.

IBGE. Instituto Brasileiro de Geografia e Estatística. Divisão regional do Brasil em regiões geográficas imediatas e regiões geográficas intermediárias. Rio de Janeiro: IBGE, 2017.

ISAAC, V. J. A.; MILSTEIN, A.; RUFFINO M. L.. A pesca artesanal no Baixo Amazonas: análise multivariada da captura por espécie. Acta Amazonica, v.26, p.185-208, 1996.

ISAAC, V. J.; CERDEIRA, R. G. P.. Avaliação e monitoramento de impacto dos acordos de pesca: região do médio Amazonas. Manaus: IBAMA, 2004.

ISAAC, V. J.; SANTO, R. E.; ALMEIDA, M. C.; ALMEIDA, O.; ROMAN, A. P.; NUNES, L.. Diagnóstico, tendência, potencial e política pública para o desenvolvimento do setor pesqueiro artesanal. Belém: SEPA, 2008.

LONGUINE, R.; SATO, L. S.; PEREIRA, M. M.; DALBELLO, J.; HÁ, N.; TAKAHASHI, L. S.. Educando hoje para pescar 
sempre. In: CONGRESSO DE EXTENSÃO UNIVERSITÁRIA DA UNESP, 6. Anais. Águas de Lindóia: UNESP, 2011.

MAIA, M. B. R.. Do defeso ao seguro desemprego do pescador artesanal: a inclusão do pescador nas políticas públicas de seguridade social. Manaus: UFAM, 2009.

NAKAÚTH, A. C. S. S.; NAKAUTH, R. F.. Programa 'Produzindo Peixe no Alto Solimões: desafios ao desenvolvimento da pesca e piscicultura'. In: ENCONTRO NACIONAL DOS NÚCLEOS DE PESQUISA APLICADA EM PESCA E AQUICULTURA, 4. Anais. Foz do Iguaçu: 2012.

PAULO-JÚNIOR, E. P. N.; XAVIER, J. H. M.; SASSI, R.; ROSA, R. S.. Gestão da pesca artesanal na Costa da Paraíba, Brasil: uma abordagem utilizando o Processo Analítico Hierárquico. Journal of Integrated Coastal Zone Management, v.12, n.4, p.509-520, 2012.

PETRERE, M.. Pesca e esforço de pesca no estado do Amazonas. Locais e aparelhos de captura e estatística de desembarque. Acta Amazonica, v.8, n.2, p.1-54, 1978.

RUFFINO, M. L.; ISAAC, V. J.. Ciclo reprodutivo de algumas espécies de peixes comerciais do Baixo Amazonas. Brasília: IBAMA, 2000.

RUFFINO, M. L.; ISAAC, V. J.. The fisheries of the Lower Amazon: questions of management and development. Acta Biologica Venezuelica, v.15, n.2, p.37-46, 1994.
RUFFINO, M. L.; OLIVEIRA, C.; VIANA, J. P.; BARTHEM, R. B.; BATISTA, V.; ISAAC, V. J.. Estatística Pesqueira do Amazonas e Pará: 2001. Manaus: IBAMA, 2002.

RUFFINO, M. L.; OLIVEIRA, C.; VIANA, J. P.; BARTHEM, R. B.; BATISTA, V.; ISAAC, V. J.. A Pesca e os Recursos Pesqueiros na Amazônia Brasileira. Manaus: IBAMA, 2004.

RUFFINO, M. L.; SOARES, E. C. S.; LOPES-JUNIOR, U.; ESTUPINÃN, G.; FONSECA, S. N.; PINTO, W. H.; OLIVEIRA, C.; BARTHEM, R. B.; BATISTA, V.; ISAAC, V. J.. Estatística Pesqueira do Amazonas e Pará: 2002. Manaus: IBAMA, 2005.

RUFFINO, M. L.; SOARES, E. C. S.; LOPES-JUNIOR, U.; ESTUPINÃN, G.; FONSECA, S. N.; PINTO, W. H.; OLIVEIRA, C.; BARTHEM, R. B.; BATISTA, V.. Estatística Pesqueira do Amazonas e Pará: 2003. Manaus: IBAMA, 2006.

SÃO PAULO. SEMA. Legislação de Pesca incidente do estado de São Paulo. São Paulo: SEMA, 2012.

SCHMITT, J.. Crime sem castigo: a efetividade da fiscalização ambiental para o controle do desmatamento ilegal na Amazônia. Tese (Doutorado) - Universidade de Brasília, Brasília, 2015.

THOMÉ-SOUZA, M. O. J. F.. Estatística Pesqueira do Amazonas e Pará: 2004. Manaus: IBAMA, 2007. ZAR, J. H.. Biostatystical Analisys. New York: Prentice-Hall, 1999.

A CBPC - Companhia Brasileira de Produção Científica (CNPJ: 11.221.422/0001-03) detém os direitos materiais desta publicação. Os direitos referem-se à publicação do trabalho em qualquer parte do mundo, incluindo os direitos às renovações, expansões e disseminações da contribuição, bem como outros direitos subsidiários. Todos os trabalhos publicados eletronicamente poderão posteriormente ser publicados em coletâneas impressas sob coordenação da Sustenere Publishing, da Companhia Brasileira de Produção Científica e seus parceiros autorizados. Os (as) autores (as) preservam os direitos autorais, mas não têm permissão para a publicação da contribuição em outro meio, impresso ou digital, em português ou em tradução. 